\title{
A Novel PMSM Hybrid Sensorless Control Strategy for EV Applications Based on PLL and HFI
}

\author{
E. Trancho ${ }^{1}$, E. Ibarra ${ }^{1}$, A. $\operatorname{Arias}^{2}$, C. Salazar ${ }^{1}$, I. Lopez $^{1}$, A. Diaz de Guereñu ${ }^{1}$, A. Peña ${ }^{1}$ \\ ${ }^{1}$ Tecnalia Research and Innovation, Industry and Transport Unit \\ Parque Científico y Tecnológico de Bizkaia, c/ Geldo, Edif. 700, 48160 Derio (Spain) \\ ${ }^{2}$ Institut d'Organització i Control, Universitat Politècnica de Catalunya. \\ Diagonal, 647, 08028 Barcelona \\ elena.trancho@tecnalia.com
}

\begin{abstract}
In this paper, a novel hybrid sensorless control strategy for Permanent Magnet Synchronous Machine (PMSM) drives applied to Electric Vehicles (EV) is presented. This sensorless strategy covers the EV full speed range and also has speed reversal capability. It combines a High Frequency Injection (HFI) technique for low and zero speeds, and a Phase-Locked Loop (PLL) for the medium and high speed regions. A solution to achieve smooth transitions between the PLL and the HFI strategies is also proposed, allowing to correctly detect the rotor position polarity when HFI takes part. Wide speed and torque four-quadrant simulation results are provided, which validate the proposed sensorless strategy for being further implemented in $\mathbf{E V}$.
\end{abstract}

\section{INTRODUCTION}

Nowadays, Permanent Magnet Synchronous Machines (PMSM) are receiving a considerable attention in a number of high performance applications, such as in electric vehicle (EV) applications, due to their high power density, high efficiency and simple structure [1]-[6].

The control of AC machines requires rotor position sensors (i.e., optical encoders or resolvers) which bring not only a higher cost but also a size increment of the drive [3]-[9]. For this reason, extensive research has been carried out in order to develop sensorless strategies. These techniques present fault tolerant capability, specially useful for EV, assuring a smooth driving when "loss of track" (LOT) or "loss of signal" (LOS) resolver fault occurs. According to the scientific literature, two approaches seem to be preferable, depending on the required speed operating range [3], [5], [10]-[12]:

a) Observer-based estimators. This kind of techniques are based on the estimation of the back-electromotive force (Back-EMF) voltage. Several approaches have been reported in the literature, such as:

- Sliding Mode Observer (SMO) based solutions [5], [13].

- Kalman Filter and Extended Kalman Filter (EKF) solutions based on state observers [14]-[16].

- Phase-Lock Loop (PLL) based solutions [4], [6], [17].

Among them, EKF based strategies are one of the most common solutions that can be found in the scientific literature for sensorless control of PMSMs. The major drawbacks of Kalman filters are the tuning of the covariance matrices appearing in their equations [14], [18] and the high execution time required during the state space estimation process [19].

Additionally, as observer-based estimators depend on the back-EMF magnitude (which is proportional to the rotor speed), these techniques fail at low speed and standstill. Thus, they are feasible only for medium to high speed ranges [7], [9], [20].

b) Signal injection based techniques. Signal injection techniques are magnetic (for IPMSM) or saturation (for SMPMSM) saliency based estimators. These methods are based on injecting high frequency test signals into the machine terminals in order to detect the rotor's position, and are suitable for zero and low speed operations [7], [9]-[11], [21]-[24]. However, the following points must be taken into account [25]:

- As HF perturbation is added to the machine, torque ripple and power losses increase. Thus, the use of such HFI technique should be minimized.

- Noise appearance, since the HFI is within the range of audible frequencies.

- The filtering steps of the HFI introduce some phase delay, which must be compensated in the obtained estimated angle.

In sensorless control of EV drives, a wide speed range including very high to low speeds and standstill must be covered. An attractive solution is based on combining both observerbased estimators and HFI strategies in order to achieve fullspeed range operation. In this sense, several hybrid observers have been presented in the literature [3], [7], [11], [25]-[27].

In this paper, a novel PLL-HFI based hybrid sensorless control strategy is proposed, which overcomes the limitations of the conventional PLL-based algorithm at low and zero speeds ranges. Thanks to the usage of a PLL approach, the computational burden of the whole hybrid sensorless algorithm is reduced, making this strategy appropriate for automotive grade Digital Signal Processors (DSPs). On the other hand, solutions to guarantee a smooth transition between both sen- 
sorless strategies and to solve the PLL problems related with speed reversal [6] are included in the proposed algorithm.

This novel sensorless control strategy has been validated in $\mathrm{EV}$ full speed range for four-quadrant region operations. A detailed simulation model including all the communications and programming protocols of the EV application has been implemented. Sensorless simulation results are provided within the document in order to show the effectiveness of this novel hybrid estimator scheme.

\section{PMSM FUNDAMENTALS}

Without considering magnetic saturation effects, the stator voltage equations of a surface mounted PMSM in the $\alpha \beta$ plane can be described as:

$$
L_{s} \frac{d \mathbf{i}_{\alpha \beta}}{d t}=-R_{s} \mathbf{i}_{\alpha \beta}+\mathbf{v}_{\alpha \beta}-\mathbf{e}_{\alpha \beta},
$$

being:

$$
\begin{gathered}
e_{\alpha}=-w_{e} \Psi_{p m} \sin \left(\theta_{e}\right), \\
e_{\beta}=w_{e} \Psi_{p m} \cos \left(\theta_{e}\right) .
\end{gathered}
$$

where $\mathbf{v}_{\alpha \beta}=\left[\begin{array}{ll}v_{\alpha} & v_{\beta}\end{array}\right]^{T}$ is the motor voltage vector, $\mathbf{i}_{\alpha \beta}=\left[\begin{array}{ll}i_{\alpha} & i_{\beta}\end{array}\right]^{T}$ is the stator current vector, $R_{s}$ is the stator resistance, $L_{s}$ is the stator inductance, $\Psi_{p m}$ is the rotor magnetic flux, and $w_{e}$ and $\theta_{e}$ are the electrical rotor speed and position, respectively.

The value of the produced electromagnetic torque in the $\alpha \beta$ reference frame can be described using the following expression:

$$
T_{e}=\frac{3}{2} P\left[\Psi_{\alpha} i_{\beta}-\Psi_{\beta} i_{\alpha}\right] .
$$

being $\Psi_{\alpha}$ and $\Psi_{\beta}$ the instantaneous value of the magnetic flux linkage in the $\alpha \beta$ reference frame and being $P$ the pole-pair number.

\section{Proposed Hybrid SEnsorless Structure}

\section{A. Introduction}

In this section, the proposed novel hybrid sensorless strategy is presented. First of all, the fundamentals of the HFI and PLLbased strategies used to cover the whole EV speed range are explained. Additionally, the proposed PLL to HFI transition strategy, which allows a reliable and smooth transition between the aforementioned strategies, is presented.

\section{B. High Frequency Injection (HFI)}

High frequency signal injection techniques use either the magnetic or saturation saliency of the machine to extract the information required for rotor position estimation. The position dependant inductance causes modulation in the resulting HF current that contains position information. These methods are not back-EMF dependant; thus, they are suitable for zero and low speed operation [7], [9]-[11], [23].
In order to extract the position information, the HF rotating voltage of (5) is added to the stator voltages calculated by the torque control algorithm [10]:

$$
\mathbf{v}_{H F}=\left[\begin{array}{l}
v_{\alpha} \\
v_{\beta}
\end{array}\right]=V_{H F}\left[\begin{array}{c}
-\sin \left(w_{H F} t\right) \\
\cos \left(w_{H F} t\right)
\end{array}\right] .
$$

The HF currents induced in the stator windings are composed by a negative and a positive sequence component as shown in (6):

$$
\mathbf{i}_{H F}=\left[\begin{array}{l}
i_{\alpha} \\
i_{\beta}
\end{array}\right]=\left[\begin{array}{l}
I_{0} \cos \left(w_{H F} t\right)+I_{1} \cos \left(2 \theta_{r}-w_{H F} t\right) \\
I_{0} \sin \left(w_{H F} t\right)+I_{1} \sin \left(2 \theta_{r}-w_{H F} t\right)
\end{array}\right] .
$$

In equation (6) it can be seen that only negative sequence component contains position information. Thus, the measured currents need to be correctly processed in order to obtain the rotor position (figure 1). The four steps to be followed in order to extract the rotor position information from the measured currents are:

a) A band pass filter is added to remove the fundamental component.

b) A coordinates rotation separates the $\mathrm{HF}$ positive and negative components. Hence, the positive sequence currents are moved down to DC while the negative sequence components are moved to twice the HF.

c) A high-pass filter is used to remove the positive sequence components now in DC from the negative sequence one.

d) A back rotation to the synchronous frame with the negative sequence obtains the following current components, which contain the position information:

$$
\left[\begin{array}{c}
i_{\alpha} \\
i_{\beta}
\end{array}\right]=I_{H F}\left[\begin{array}{l}
\cos \left(2 \theta_{e}\right) \\
\sin \left(2 \theta_{e}\right)
\end{array}\right] \text {. }
$$

The angle $2 \theta$ needs to be restored to $\theta$, ensuring that the estimated angle is checked for the correct polarity. The latter is needed because signal injection techniques cannot distinguish between the north and south poles of the rotor magnetic field. In this sense, a strategy which overcomes this limitation is presented in this paper in section III-D.

\section{Phase-Locked Loop (PLL)}

PLL control strategies have been traditionally used for power converter synchronization with the power grid [28][30]. However, it is also possible to use PLL structures for sensorless rotor position and speed estimation of electrical machines [4].

In the proposed hybrid sensorless solution, the PLL structure of figure 2 is used to operate on the back-EMF $\alpha \beta$ components (taken as input signals).

From (2) and (3) the estimated back-EMF can be expressed as:

$$
\begin{gathered}
\widehat{e}_{\alpha}=-\Psi_{p m} \widehat{w}_{e} \sin \left(\widehat{\theta}_{e}\right), \\
\widehat{e}_{\beta}=\Psi_{p m} \widehat{w}_{e} \cos \left(\widehat{\theta}_{e}\right),
\end{gathered}
$$

being 


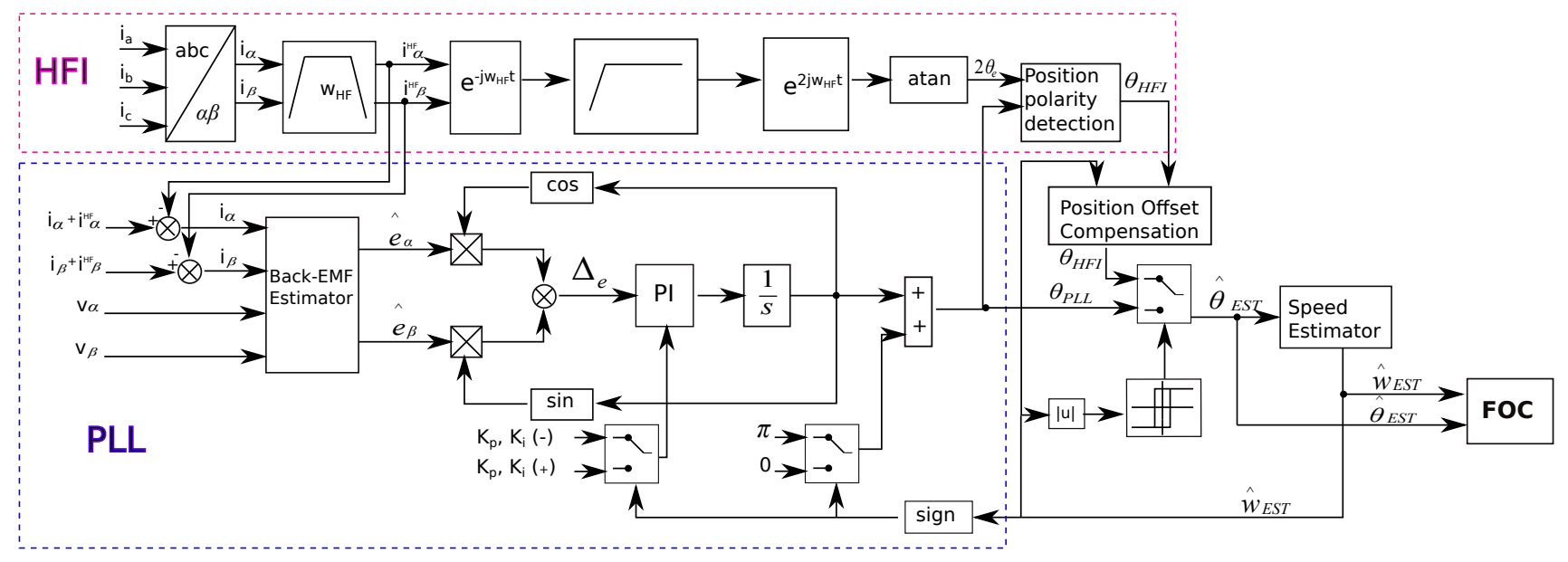

Fig. 3. Hybrid angle estimation block diagram with the transition strategy between the PLL and HFI.

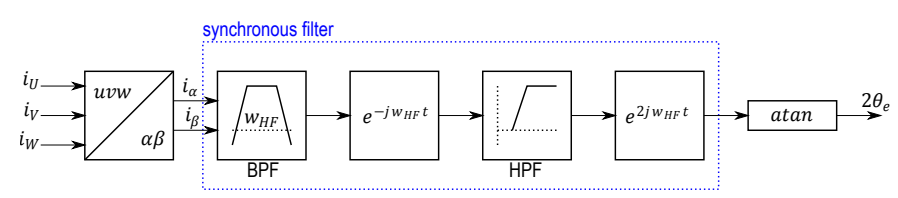

Fig. 1. HFI algorithm angle estimation block diagram.

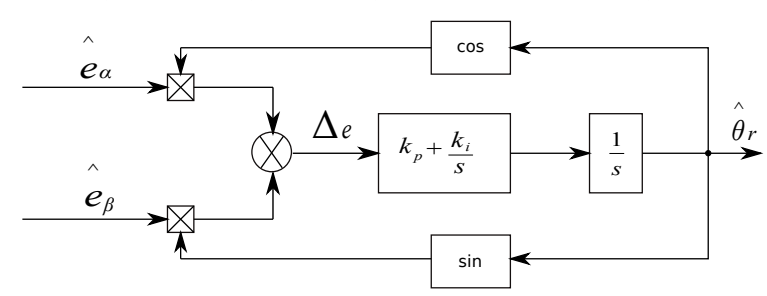

Fig. 2. PLL-based rotor position estimation diagram.

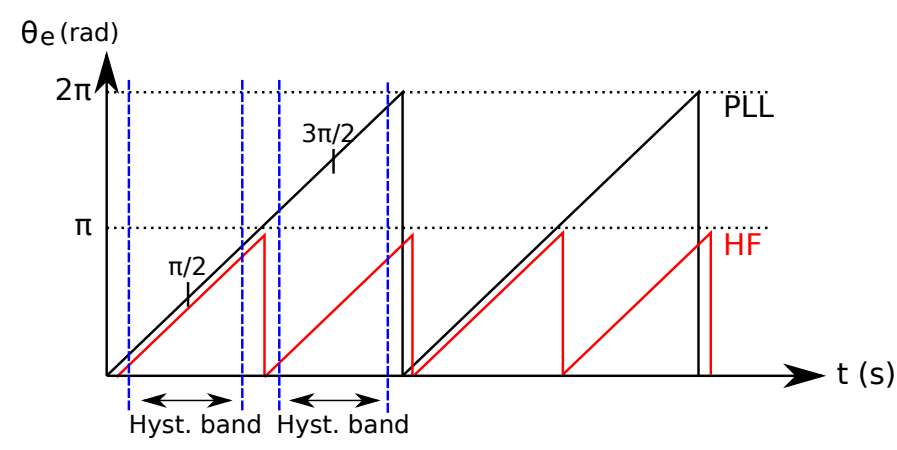

Fig. 4. Flag initialization hysteresis bands.

$$
\widehat{\theta}_{e}=\widehat{\theta}_{r}+\theta_{\text {error }}
$$

Operating (8) and (9), the following expression is obtained:

$$
-\widehat{e}_{\alpha} \cos \left(\widehat{\theta}_{r}\right)-\widehat{e}_{\beta} \sin \left(\widehat{\theta}_{r}\right)=\widehat{w}_{e} \Psi_{p m} \theta_{\text {error }}
$$

where $\widehat{\theta}_{e}$ and $\widehat{w}_{e}$ are the rotor position and speed, respectively (both included in the estimated back-EMF), $\hat{\theta}_{r}$ is the estimated rotor position, and $\theta_{\text {error }}$ is the angle deviation.

The Proportional Integral (PI) regulator is used to correct the angle deviation, bringing the estimated angle to converge to the reference one. Conventional PLL based sensorless strategies fail when rotation direction changes due to the following reasons [6]:

- The PI regulator $K_{p}$ and $K_{i}$ gains calculated for positive speed operation are not valid for negative speed operation. Thus, these gains must be adapted for each operation condition, which is generally a complex task.

- An offset of $180^{\circ}$ is obtained in the estimated angle when the speed is negative.

In order to overcome these problems, a novel PLL scheme has been designed, where PI regulator coefficients are scheduled as a function of the estimated speed sign in order to correctly operate in full speed range. When speed reversal happens, the sign of the back-EMF changes, driving the PLL to converge with a $\pi$ rad offset. Thus, angle offset correction has been taken into account when designing the proposed hybrid structure, assuring robustness against speed reversals. This PLL reconfiguration is performed without adding any disturbance to angle and speed estimation. This is achieved because the angle estimation is performed by HFI during the aforementioned reconfiguration procedure.

The proposed transition strategy is thoroughly explained in the following subsection.

\section{Proposed PLL-to-HFI transition strategy}

Figure 3 shows the general diagram of the proposed hybrid angle and speed estimation algorithm. Each time signal injection is activated, initial rotor position polarity detection must be checked in order to restore $\theta$ from $2 \theta$. Otherwise, the estimated angle could be $\pi$ rad off from the actual rotor angle. The strategy proposed consists on determining whether or not it is necessary to add an offset of $\pi$ rad to the rotor position, as shown in figure 4 . 


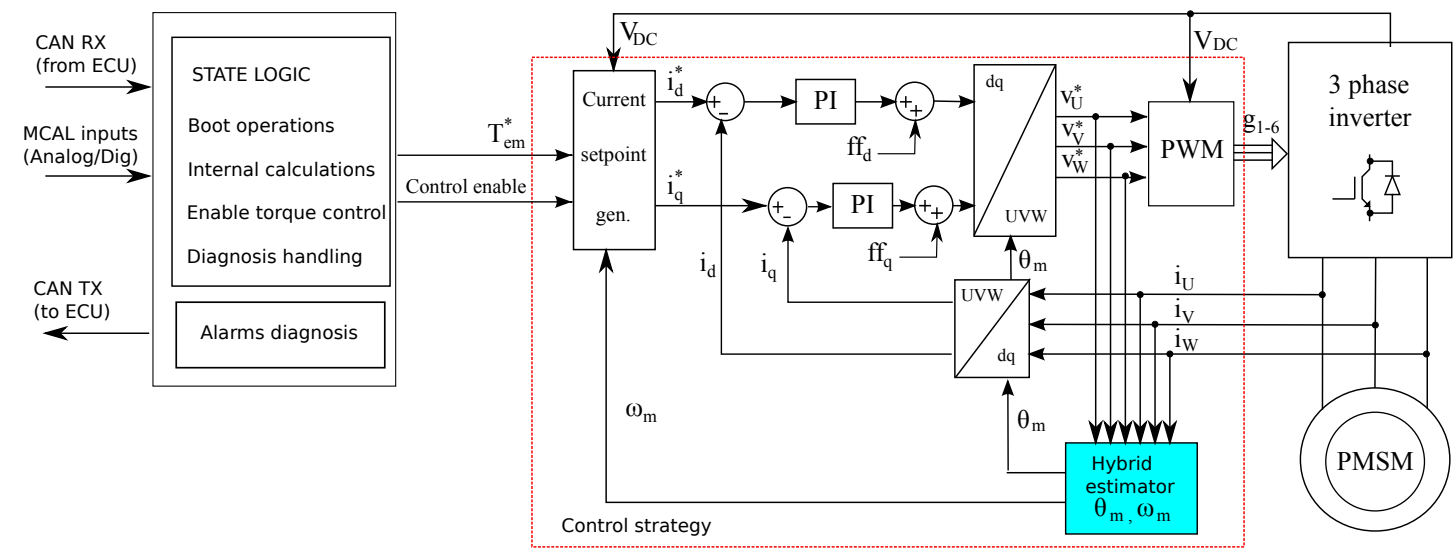

Fig. 6. Implemented EV sensorless control strategy with the hybrid speed and angle estimator.

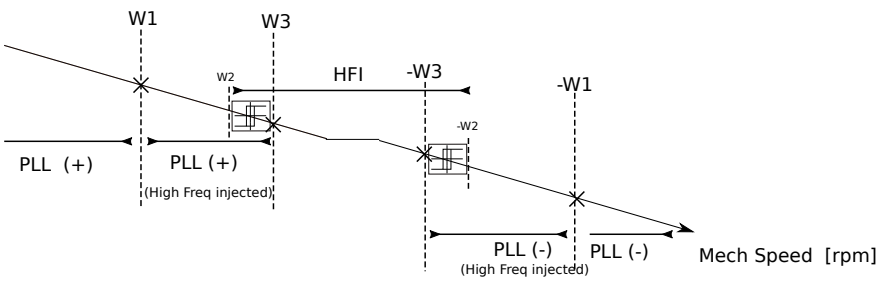

Fig. 5. Hybrid sensorless control strategy along full speed range
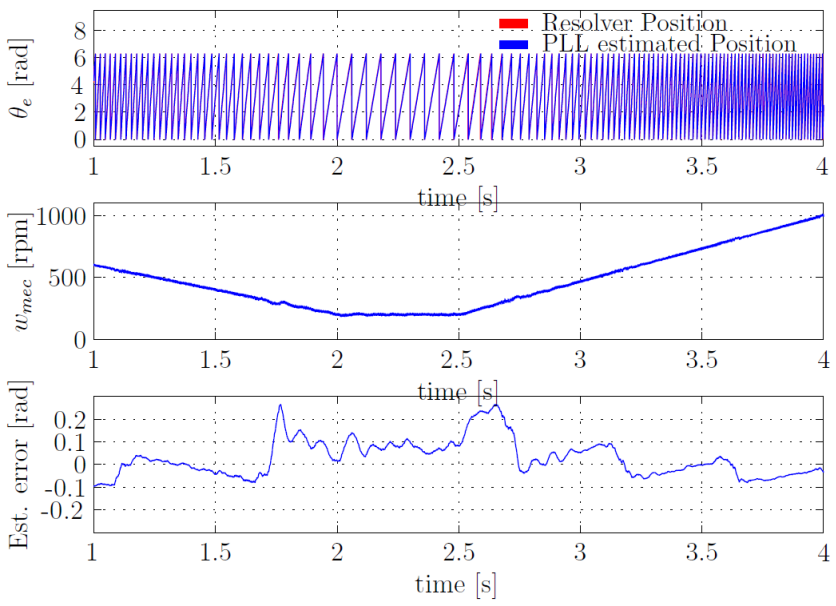

Fig. 7. PLL sensorless simulation results.

The initial position polarity detection strategy is based on the information obtained from the estimated position by the PLL structure. The calculator determines the PLL estimated position polarity, establishing thus the angle offset to be added to the HFI estimated angle, as follows:

$$
\text { Offset }= \begin{cases}0 & \text { if } \theta_{P L L}=\frac{\pi}{2} \pm \text { HystBand } \\ \pi & \text { if } \theta_{P L L}=\frac{3 \pi}{2} \pm \text { HystBand }\end{cases}
$$

An hysteresis band has been added for the polarity detection (figure 4) for the following reasons:
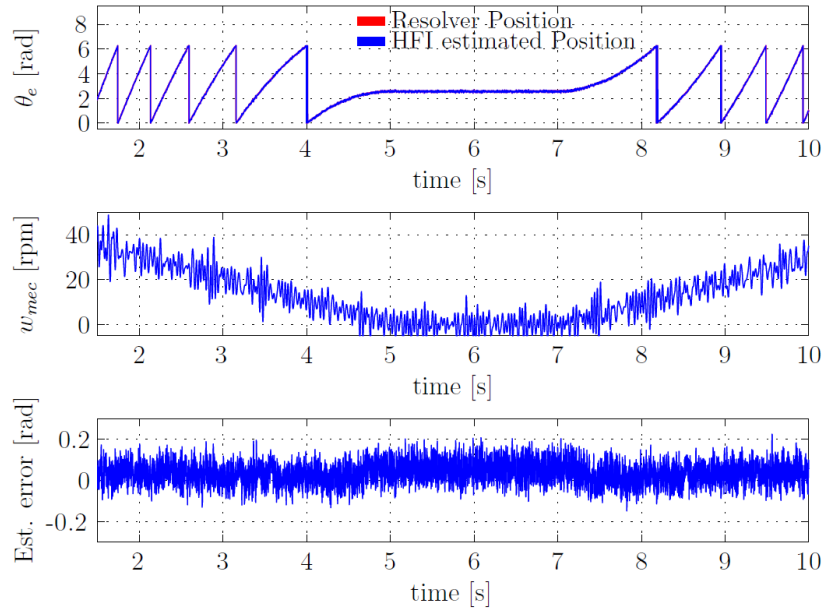

Fig. 8. HFI sensorless simulation results.

(a) Errors in the polarity determination due to possible angle offsets between both estimators must be avoided.

(b) The robustness of the strategy against electromagnetic noise induced in the measured electrical magnitudes must be enough for the given application.

Hence, a robust position polarity detection and, consequently, a reliable change between PLL and HFI modes is ensured. The width of the hysteresis band must be adjusted depending on the aforementioned factors.

The polarity estimation performed by the PLL is robust, because there is enough back-EMF for the PLL during the speed range in which the polarity determination is carried out (PLL + High frequency injected speed range, figure 5).

A speed dependant look-up-table is included to compensate the position offset due to the filters when using HFI. This strategy calculates offline the delay introduced by the aforementioned filters and the position error is mapped for all the HFI operating points.

The general transition process is shown in figure 5. The hybrid sensorless controller uses the estimated rotor position 

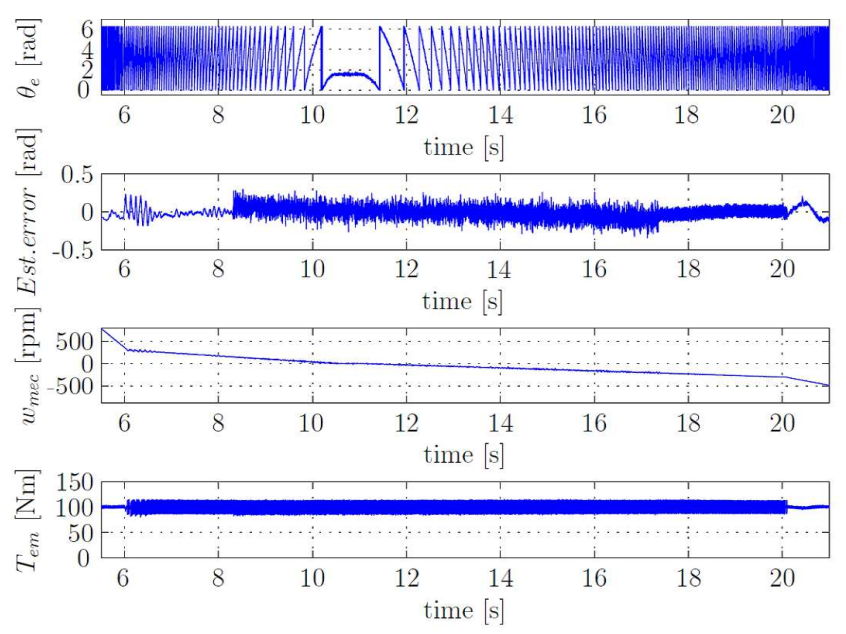

Fig. 9. Hybrid sensorless simulation results for nominal torque.

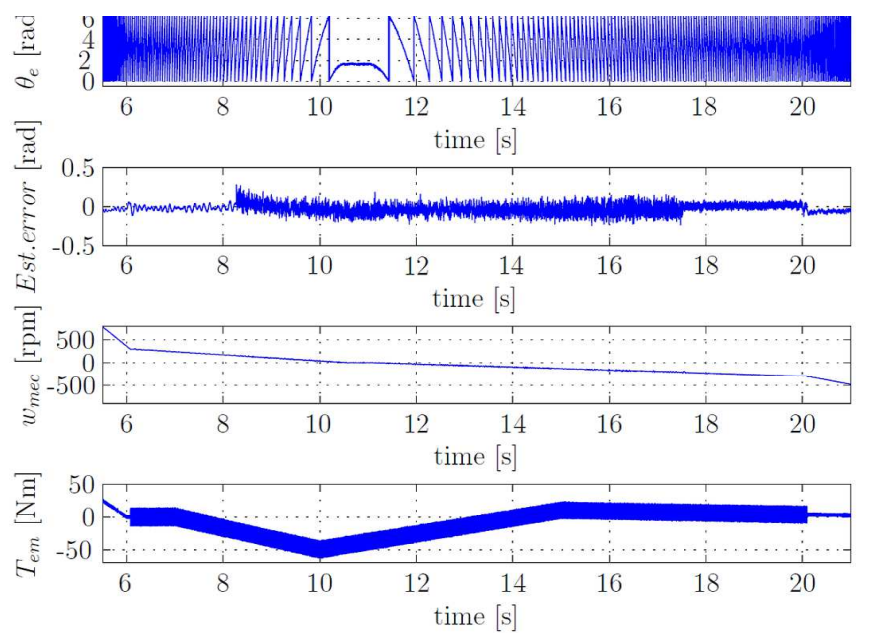

Fig. 10. Hybrid sensorless simulation results in four quadrants operation.

obtained with HFI from zero speed to a given speed. Beyond this speed, the angle estimated by the PLL strategy is selected. The HF voltage necessary for the HFI method is not turned off until a speed threshold is reached, where the PLL estimation is used for feedback. Once the signal injection voltage is turned off, the HFI position estimation becomes very inaccurate. This speed threshold is required to assure estimation convergence. Using this strategy, the transition between both estimations is correctly performed for any speed and acceleration profiles.

Once $\theta_{H F I}$ and $\theta_{P L L}$ are determined, the transition between both observers is carried out (figure 3). There are different possibilities to realize the transition, among which the most common is the use of weighting coefficients [11]. In this paper, a speed dependant switch including a hysteresis band is proposed in order to avoid position signal degradation when operating at the speed threshold. This way, robustness and reliability are ensured when the transition occurs.

Finally, $\widehat{w}_{E S T}$ is obtained from the final estimated ro- tor position $\widehat{\theta}_{E S T}$. When the PLL strategy is active (figure 5 ), the estimated speed is not obtained directly from the PLL. This is due to the fact that HF components appear in the aforementioned estimated speed when PLL and HFI estimations coexist (figure 5). When estimating the angle, the PLL integrator minimizes the HF component present in the estimated speed, because the average value of this HF component is zero. In this way, the usage of filters is reduced, reducing the overall computational burden of the sensorless algorithm. Another optimisation employed to reduce the use of additional filters is the subtraction of the current high frequency component from the PLL's current inputs (figure 3). In this way, the perturbations produced by the HF into the back-EMF estimation are partially reduced.

\section{Sensorless Control Results}

In order to validate the proposed hybrid sensorless strategy, an automotive PMSM drive system (one single motor connected to the transmission axis throughout a mechanical coupling) has been simulated in Matlab/Simulink, including the sensorless strategy of figure 3. A conventional Field Oriented Control (FOC), covering the field weakening region, has been designed for full speed range torque control (see figure 6). A Model in the Loop (MiL) approach has been followed, taking care of the communications and programming protocols of the EV industry, as well as including a detailed power electronics and an electrical PMSM machine model. The motor under test is an automotive SM-PMSM with a maximum speed of $8000 \mathrm{rpm}$ and a nominal power of $64 \mathrm{~kW}$, being the nominal inductances in the $d q$ axis $L_{d}=0.292 \mathrm{mH}$ and $L_{q}=0.292 \mathrm{mH}$ (with a level of saliency of $10 \%$ ), $R_{s}=19.1 \mathrm{~m} \Omega$ and $\Psi_{p m}=0.0731 \mathrm{~Wb}$. The machine employed in this particular case is highly linear, thus the magnetic saturation is negligeable.

Figures 7 and 8 show the simulation results obtained respectively for the PLL and HFI strategies. In figure, 7 the results of the measured resolver position $\theta_{r}$ versus the estimated position $\widehat{\theta}_{e}$ and the estimated rotor speed $\widehat{w}_{r p m}$ using PLL method are presented, verifying its correct performance from medium to high speed regions. On the other hand, figure 8 shows the results obtained for the HFI strategy at very low and zero speeds. As it can be seen from figure 8, the HFI technique is able to track the angle up to standstill certainly. Both sensorless introduce an error of the electrical angle within the range of $\pm 0.2 \mathrm{rad}$, which is an acceptable figure for closing the loop (figures 7 and 8).

Finally, figure 9 and figure 10 show the simulation results of the hybrid sensorless strategy proposed for a wide speed range, including standstill and speed reversals, commanding a nominal torque reference of $100 \mathrm{Nm}$ (figure 9) and in four quadrant operation (figure 10). As it can be seen from the results, the PLL to HFI transition is smoothly carried out, following the transition criteria described in figure 5. In this practical case, the speed thresholds has been set as: $w_{1}=$ $300 \mathrm{rpm}, w_{2}=225 \mathrm{rpm}, w_{3}=150$. Additional torque ripple appears in figure 9, produced by the high frequency signal 
injected in the intervals $\left[\left|w_{1}\right|-\left|w_{3}\right|\right]$, and consequently the power losses of the system increases. However, due to the high inertia of the EV, this ripple does not denote any problem to the vehicle dynamics.

\section{CONCLUSIONS}

A hybrid sensorless field-oriented control has been proposed for four-quadrant Electric Vehicles drives equipped with permanent magnet synchronous machines. The proposed hybrid sensorless algorithm estimates the angle from a PLL, for medium and high speeds, together with a non-model-based voltage HF injection technique, which only works at zero and low speeds. Solutions to overcome PLL to HFI transition limitations are proposed, obtaining a full speed sensorless control suitable for EV applications.

In real automotive applications, the whole control algorithm must be executed at a very low sample time (in this application $80 \mu \mathrm{s}$ at most). The use of a PLL instead of observer-based techniques such as Kalman filters requires less computational capability and therefore, the CPU demand for the sensorless strategy is reduced, which is always a merit for EV industry.

An accurate model has been developed to validate the proposed strategy when speed reversal is performed for wide torque levels. Such accurate model considers not only the machine's saliency, the real plant delays and the proposed hybrid sensorless field-oriented control, but also all the communications and programming protocols of the EV real application. Electrical angle errors are kept within the range of $+/-0.2 \mathrm{rad}$, which is a promising figure for facing the next experimental implementation.

\section{ACKNOWLEDGMENT}

The present work is partially supported by the Basque Goverment under the projects KT4eTRANS (ELKARTEK program KK-2015/00047), FPGAmc (EMAITEK program) and by the spanish projects DPI2013-41224-P (Ministerio de Educación) and 2014 SGR 267 (AGAUR).

\section{REFERENCES}

[1] B. Asaei and B. Rahrovi, "Minimum-copper-loss control over full speed range of an ipmsm drive for hybrid electric vehicle application," in IEEE Vehicle Power and Propulsion Conference (VPPC), 2010.

[2] T. Finken, M. Hombitzer, and K. Hameyer, "Study and comparison of several permanent-magnet excited rotor types regarding their applicability in electric vehicles," in Emobility - Electrical Power Train Conference, 2010

[3] R. P. F. Parasiliti and M. Tursini, "Sensorless speed control of a pm synchronous motor based on sliding mode observer and extended kalman filter," IEEE, 2001.

[4] R. Li and G. Zhao, "Position sensorless control for pmsm using sliding mode observer and phase-locked loop," in Proc. of the Power Electronics and Motion Control Conference, 2009.

[5] S. Chi and L. Xu, "Position sensorless control of pmsm based on a novel sliding mode observer over wide speed range," in Proc. of the Power Electronics and Motion Control Conference, 2006.

[6] C. Olivieri and M. Tursini, "Anovel pll scheme for a sensorless pmsm drive overcoover common speed reversal problems," in Pro. of the International Symposium on Power Electronics, Electrical Drives, Automation and Motion, 2012.

[7] A. Piipo, M. Hinkkanen, and J. Luomi, "Sensorless control of pmsm drives using a combination of voltage model and hf signal injection," in Proc. of the IEEE Industry Applications Conference, 2004.
[8] Z. Qiao, T. Shi, Y. Wang, Y. Yan, C. Xia, and H. X., "New slidingmode observer for position sensorless control of permanent-magnet synchronous motor," IEEE Transactions on Industrial Electronics, vol. 60, no. 2, pp. 710-719, 2013.

[9] J. H. Jang, S. K. Sul, J. I. Ha, and K. Ide, "Sensorless drive of surface-mounted permanent-magnet motor by high-frequency signal injection based on magnetic saliency," IEEE Transactions on Industry Applications, vol. 39, no. 4, pp. 1031-1039, 2003.

[10] A. Arias, G. Asher, M. Sumner, P. Wheeler, L. Empringham, and C. Silva, "High frequency voltage injection for the sensorless control of permanent magnet synchronous motors using matrix converters," in Proc. of the IEEE Industrial Electronics Conference (IECON), 2004.

[11] I. Omrane, W. Dib, E. Etien, and O. Bachelier, "Sensorless control of pmsm based on a nonlinear observer and a high-frequency signal inj ection for automotive applications," IEEE, pp. 3130-3135, 2013.

[12] Y. Lee, Y. Kwon, and S. Sul, "Comparison of rotor position estimation performance in fundamental-model-based sensorless control of pmsm," in Proc. of the IEEE Energy Conversion Congress and Exposition (ECCE), 2015, pp. 5624-5633.

[13] S. Chen, Y. Luo, and Y. Pi, "Pmsm sensorless control with separate control strategies and smooth switch from low speed to high speed," ISA Transactions, vol. 58, pp. 650-658, 2015.

[14] S. Bolognani, L. Tubiana, and M. Zigliotto, "Extended kalman filter tuning in sensorless pmsm drives," IEEE Transactions on Industry Applications, vol. 39, no. 6, pp. 1741-1747, 2003.

[15] D. Janiszewski, Extended Kalman Filter Based Speed Sensorless PMSM Control with Load Reconstruction. INTECH, 2010.

[16] V. Bindu, A. Unnikrishnan, and R. Gopikakumari, "Sensor less vector control of pmsm using a blind system identification approach based on extended kalman filter algorithm," in IEEE International Conference on Computational Intelligence and Computing Research, 2010.

[17] G. El-Murr, D. Giaouris, and J. Finch, "Universal pll strategy for sensorless speed and position estimation of pmsm," in Proc. of the IEEE Industrial and Information Systems Conference, 2008.

[18] M. Huang, A. Moses, and F. Anayi, "The comparison of sensorless estimation techniques for pmsm between extended kalman filter and flux-linkage observer," in Applied Power Electronics Conference and Exposition (APEC), 2006, pp. 654-659.

[19] A. M. L. Idkhajine, E. Monmasson, "Extended kalman filter for ac drive sensorless speed controller - fpga-based solution or dsp-based solution," IEEE, 2010.

[20] S. Zaim, B. Nahid-Mobarakeh, and F. Meibody-Tabar, "Robust position sensorless control of nonsalient pmsm at standstill and low speeds," IEEE Journal of Emerging and Selected Topics in Power Electronics, vol. 2, no. 3, pp. 640-650, 2014.

[21] R. Leidhold, "Position sensorless control of pm synchronous motors based on zero-sequence carrier injection," IEEE Transactions on Industr, 2011.

[22] M. C. and. D. Lorenz, "Rotor position and velocity estimation for a salient-pole permanent magnet synchronous machine at standstill and high speeds," IEEE Transactions on Industrial Applications, 1998.

[23] B. Liu, B. Zhou, J. Wei, H. Liu, J. Li, and L. Wang, "A rotor initial position estimation method for sensorless control of spmsm," in Proc. of the Industrial Electronics Conference, 2014, pp. 354-359.

[24] W. Chi, M. Cheng, C. Li, K. Su, and W. Li, "Motor parameter autoidentification based on sensorless startup procedure for pmsm drives," in Proc. of the Industrial Electronics Conference (IECON), 2014, pp. 3861-3866.

[25] G. A. C. Silva and M. Summer, "Hybrid rotor position observer for wide speed-range sensorless pm motor drives including zero speed," IEEE Transactions on Industrial Electronics, 2006.

[26] E. F. S. Bolognani, A. Faggion and L. Sgarbossa, "Full speed range sensorless ipm motor drives," 2012.

[27] J. Z. J. E. J. P. A. Arias ., C. Ortega, "Hybrid sensorless permanent magnet synchronous machine four quadrant drive based on direct matrix converter," in Electrical Power and Energy Systems, 2012.

[28] V. B. V. Kaura, "Operation of a phase locked loop system under distorted utility conditions," IEEE Transactions on industry applications, vol. 33, no. 1 , pp. $58-63$, January 1997.

[29] M. R. I. M., Karimi-Ghartemani, "A method for synchronization of power electronic converters in polluted and variable-frequency environments," IEEE TRANSACTIONS ON POWER SYSTEMS, 2004.

[30] S.-K. Chung, "A phase tracking system for three phase utility interface inverters," IEEE TRANSACTIONS ON POWER SYSTEMS, 2000. 